\title{
A challenging case of hereditary type 1 tyrosinaemia associated with persistent diarrhoea: Case report and literature review
}

\author{
N A Yassin, MD; E A Mogahed, MD; H El-Karaksy, MD \\ Department of Paediatrics, Qasr Al Ainy Faculty of Medicine, Cairo University, Egypt \\ Corresponding author: N A Yassin (noha.adel20@yahoo.com)
}

\begin{abstract}
Hereditary tyrosinaemia type 1 (HT1) is a rare inherited genetic disorder attributed to deficiency of the enzyme fumarylacetoacetate hydrolase (FAH). HT1 may present with diarrhoea in its acute form. We report on a 2.5-year-old Egyptian girl diagnosed with HT1. At the age of 1 year and 9 months, the patient started to have persistent diarrhoea with marked abdominal distension, anorexia and irritability, and with no fever or vomiting. At the onset of diarrhoea, liver synthetic functions deteriorated and ascites progressed, requiring frequent paracentesis. These manifestations did not improve after starting specific treatment for HT1 (2-(2-nitro-4-trifluoromethylbenzoyl)1,3-cyclohexanedione) (NTBC). Coeliac disease was diagnosed; this disorder was not previously reported as having an association with HT1.
\end{abstract}

S Afr J Child Health 2020;14(2):104-106. https://doi.org/10.7196/SAJCH.2020.v14i2.1661

Hereditary tyrosinaemia type 1 (HT1) is an inherited autosomal recessive metabolic disorder secondary to deficiency of fumarylacetoacetate hydrolase (FAH), which is a terminal enzyme in the metabolism of tyrosine. The gene for this enzyme has been mapped to the long arm of chromosome 15. While primarily synthesised in the liver, FAH is also synthesised in the kidneys, intestine, heart, adrenal glands, lungs, stomach, pancreas, lymphocytes and skeletal muscles. ${ }^{[1]}$ HT1 typically affects the liver and kidneys, and is classified into acute, subacute and chronic forms. In acute form, diarrhoea, vomiting, jaundice and acute liver failure develop early after birth. The subacute form manifests with hepatomegaly and coagulopathy, usually during infancy. The chronic form manifests after one year of age, with slowly progressive liver disease, cirrhosis and increased risk of hepatocellular carcinoma. Renal tubular dysfunction such as Fanconi syndrome appears in the acute and chronic forms. As a result of Fanconi syndrome, patients exhibit hypophosphataemic rickets. Elevation of serum alphafetoprotein is characteristic of HT1. ${ }^{[2]}$

We report here a case of HT1 associated with persistent diarrhoea showing no improvement despite starting treatment with HT1 (2-(2-nitro-4-trifluoromethylbenzoyl)-1,3-cyclohexanedione) (NTBC).

The case report was approved by the institutional review board and ethical committee of Qasr Al Ainy Faculty of Medicine, after informed consent was signed by the patient's guardians.

\footnotetext{
Case

A 2.5-year-old Egyptian girl presented to the Paediatric Hepatology Unit of Cairo University Specialised Children's Hospital at the age of 4 months with progressive abdominal distension. There was no history of jaundice, change in the colour of the urine or stools or bleeding. Abdominal examination revealed generalised abdominal distension owing to hepatosplenomegaly. There was no ascites. Other system examinations were normal.

Liver function tests revealed normal bilirubin and transaminases with impaired synthetic functions: international normalised ratio
}

(INR) 2.8, serum albumin $2.6 \mathrm{~g} / \mathrm{dL}(\mathrm{N}: 3.6-5.2)$ and very high alkaline phosphatase of $1650 \mathrm{U} / \mathrm{L}$ (N: up to $640 \mathrm{U} / \mathrm{L}$ ). Serum calcium was $9.3 \mathrm{mg} / \mathrm{dL}(\mathrm{N}: 9-11 \mathrm{mg} / \mathrm{dL})$ and serum phosphorus was low: $2.2 \mathrm{mg} / \mathrm{dL}(\mathrm{N}: 4-6.5 \mathrm{mg} / \mathrm{dL}$ ). Arterial blood gas analysis showed compensated metabolic acidosis. Alpha-fetoprotein was highly elevated: $125000 \mathrm{ng} / \mathrm{mL}$ (N: 0 - 11). Abdominal ultrasonography showed a heterogeneous liver and enlarged spleen.

HT1 was suspected and the diagnosis was confirmed by the presence of highly elevated succinyl acetone levels in a dry bloodspot $(42 \mu \mathrm{mol} / \mathrm{L})$ and urine.

A tyrosine- and phenylalanine-restricted diet was started. NTBC treatment for HT1 was not available in Egypt at the time of diagnosis and liver transplantation could not be performed because of donor unavailability.

During her follow-up, the patient developed clubbing, palmar erythema, lower limb oedema, ascites, rachitic manifestations in the form of broadening of the wrists and frontal bossing, and multiple small hepatic focal lesions, with gradual deterioration of her weight and length (-0.42 SD and $-2.15 \mathrm{SD}$, respectively). Her alphafetoprotein level was $17900 \mathrm{ng} / \mathrm{mL}$. Triphasic computed tomography (CT) scanning showed enhancing nodules with rapid wash-out. Liver biopsy was not attempted because of the increased INR. At the age of 1 year and 9 months, the patient started to experience diarrhoea (3 - 7 loose non-bloody motions/day) and marked abdominal distension, with no fever or vomiting. Since that time, she had several attacks of dehydration with metabolic acidosis and electrolyte imbalance requiring repeated hospitalisation. At the onset of diarrhoea, liver synthetic functions deteriorated and ascites became refractory to diuretic therapy and albumin infusions, requiring occasional therapeutic paracentesis. In addition, she had marked microcytic hypochromic anaemia refractory to iron therapy, for which she received packed red blood cells (RBCs) once, although there was no history of gastro-intestinal haemorrhage or bleeding from any other body orifices.

At the age of 2 years, the patient started to receive NTBC. Although urinary succinyl acetone became undetectable, the 
patient did not show any clinical improvement regarding the diarrhoea, marked abdominal distension, ascites, anaemia, hypoalbuminaemia $(1.6 \mathrm{~g} / \mathrm{dL})$ and electrolyte imbalance. Abdominal ultrasound at that time showed marked ascites and severe intestinal loop dilatation.

We started to investigate other aetiologies that may cause chronic diarrhoea. Microscopic stool examination was negative for Cryptosporidium but revealed Giardia lamblia and Helicobacter pylori antigen was positive. The patient received eradication therapy for H. pylori (pantoprazole plus amoxicillin and clarithromycin) for 1 month. Although stool analysis showed resolution of infection following therapy, the patient did not show any clinical improvement. A quantiferon test was done to exclude tuberculosis and was negative. Primary immune deficiency and HIV infection were excluded as well.

Screening for coeliac disease was done. The patient had a normal serum level of total IgA and was positive for anti-tissue transglutaminase (tTG) antibody of IgA type (46 units). Upper gastrointestinal endoscopy showed superficial erosions in the duodenal bulb. Histopathological examination of the duodenal biopsy revealed mild duodenitis with preserved villous architecture, intraepithelial lymphocytes, and mild Brunner's gland hyperplasia (Marsh grade 1) (Fig. 1). Human leukocyte antigen (HLA) typing for coeliac disease was not feasible, and hence a diagnosis of coeliac disease was suspected, a trial of gluten-free diet was applied, and the patient was then put on a strict gluten-free diet, in addition to the tyrosine- and phenylalanine-restricted diet, which was followed by marked improvement of diarrhoea within 2 weeks and abdominal distension within 1 month of the strict gluten-free diet, while anaemia, oedema, hypoalbuminaemia, ascites and elevated liver enzymes improved 6 months later (Table 1). Alpha-fetoprotein levels decreased to $120 \mathrm{ng} / \mathrm{mL}$. Succinyl acetone in the urine became undetectable. Surveillance for hepatocellular carcinoma was done using alpha-fetoprotein levels and abdominal ultrasonography every 3 months. Abdominal CT is performed annually. The family was given genetic counselling. The child is now awaiting liver transplantation.

\section{Discussion}

Chronic diarrhoea has many diverse causes. Comorbid symptoms should be considered when constructing a differential diagnosis. Misdiagnosis typically results from failure to appreciate all the available evidence and

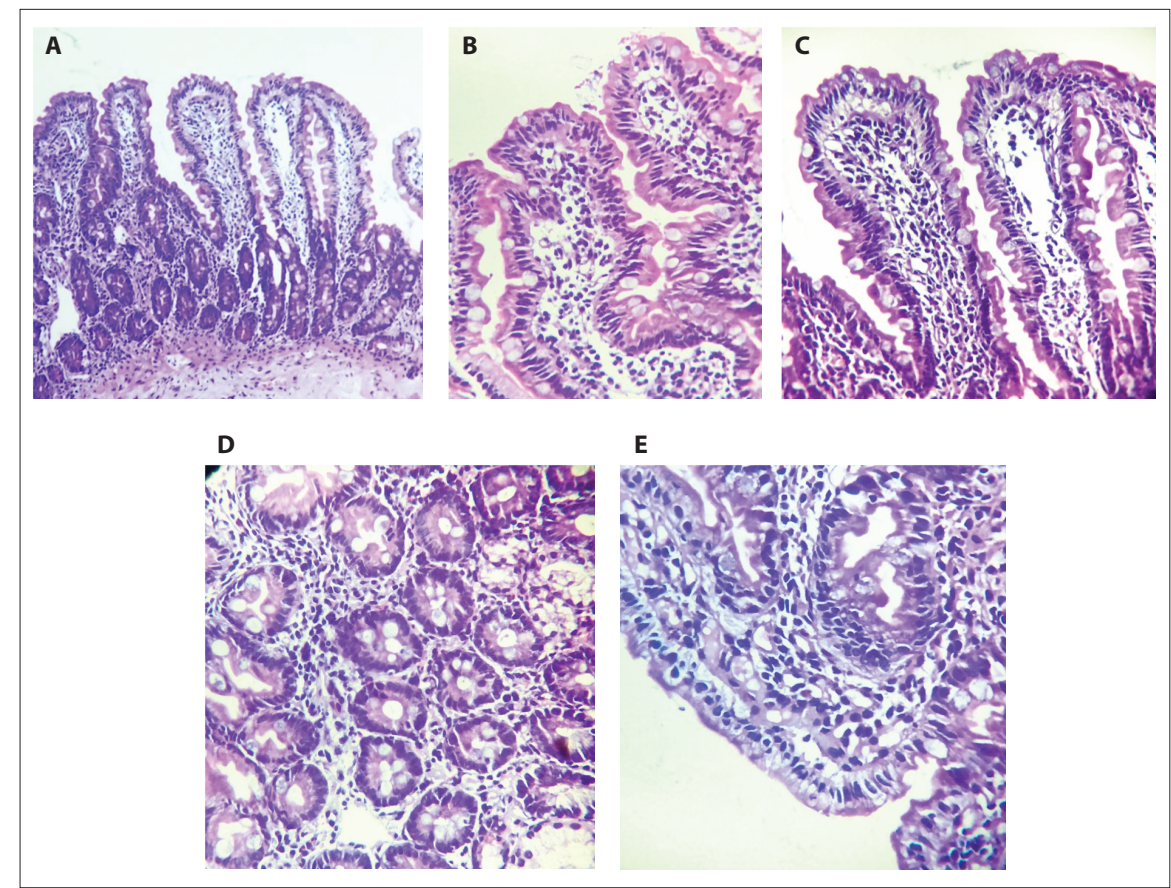

Fig. 1. Photomicrography of the duodenum. Images $A, B$ and $C$ show preserved villous patterns with focally increased intraepithelial lymphocytes (H\&E stain, original magnification $\times 10, \times 20$ and $\times 20$, respectively). Images $D$ and E show mild crypt hyperplasia and intraepithelial lymphocytes (H\&E stain, original magnification $\times 20$ ).

Table 1. Laboratory investigations before and after gluten-free (GF) diet

\begin{tabular}{|c|c|c|}
\hline Laboratory tests & $\begin{array}{l}\text { Before } \\
\text { GF diet }\end{array}$ & $\begin{array}{l}6 \text { months } \\
\text { after GF diet }\end{array}$ \\
\hline \multicolumn{3}{|l|}{ Complete blood count } \\
\hline Total leucocyte count, $\times 10^{9} / \mathrm{L}$ & 4.5 & 4.5 \\
\hline Haemoglobin, g/dL & 7.1 & 10.5 \\
\hline $\mathrm{MCV}, \mathrm{fL}$ & 70 & 70.4 \\
\hline Haematocrit, \% & 21 & 32.1 \\
\hline Platelets, $\times 10^{9} / \mathrm{L}$ & 55 & 64 \\
\hline \multicolumn{3}{|l|}{ Liver function tests } \\
\hline ALT, U/L (5 - 45) & 58 & 42 \\
\hline AST, U/L (13 - 35) & 91 & 53 \\
\hline GGT, U/L (5 - 32) & 90 & 80 \\
\hline ALP, U/L (N up to 640$)$ & 946 & 473 \\
\hline Albumin, g/dL (3.6 - 5.2) & 1.6 & 3.8 \\
\hline Total bilirubin, mg/dL $(<1.2)$ & 1.8 & 0.6 \\
\hline Direct bilirubin mg/dL $(\leq 0.2)$ & 0.7 & 0.3 \\
\hline INR & 2.9 & 1.1 \\
\hline Calcium, mg/dL (8.8 - 10.8) & 7.5 & 8.3 \\
\hline Phosphorus, mg/dL (4 - 6.5) & 2.3 & 4.8 \\
\hline Iron, $\mu \mathrm{g} / \mathrm{dL}(40-100)$ & 32 & 65 \\
\hline Tissue transglutaminase IgA antibodies (units)* & 46 & 7 \\
\hline \multicolumn{3}{|c|}{$\begin{array}{l}\mathrm{MCV}=\text { mean cell volume; } \mathrm{ALT}=\text { alanine transaminase; } \mathrm{U} / \mathrm{L}=\text { units per litre; } \mathrm{AST}=\text { aspartate aminotransfera } \\
\mathrm{GGT}=\text { gamma-glutamyl transferase; } \mathrm{ALP}=\text { alkaline phosphatase; } \mathrm{INR}=\text { international normalised ratio. } \\
{ }^{*} \mathrm{~N}<20 \text { (negative); } 20 \text { - } 30 \text { (weak positive); }>30 \text { (positive). } \\
{ }^{\mathrm{N}} \text { Negative. }\end{array}$} \\
\hline
\end{tabular}

from not considering the entire differential diagnosis of chronic diarrhoea. ${ }^{[3]}$

As far as we know, the present report is the first of a child with HT1 associated with coeliac disease. Although HT1 in its acute form can present with growth retardation, diarrhoea, vomiting and hepatic dysfunction in the first few weeks of life, ${ }^{[2]}$ our patient 
experienced diarrhoea later on, mostly after the introduction of a gluten-containing diet. Treatment with NTBC together with restriction of tyrosine and phenylalanine in the diet is usually associated with clinical improvement of HT1 and can prevent neurological crises, acute hepatic failure, and renal tubular acidosis. ${ }^{[4]}$ In the present case, the diarrhoea was erroneously attributed to HT1 - but even with full doses of NTBC, she did not show clinical improvement.

Our patient had the classic symptoms of coeliac disease (chronic diarrhoea, abdominal distension, irritability, anorexia, anaemia and growth retardation). There was an overlap of these symptoms between HT1 and coeliac disease, which led to the delayed diagnosis of the associated comorbid condition, particularly the rapidly accumulating ascites despite NTBC therapy.

Liver abnormalities are common extra-intestinal manifestations in patients with coeliac disease. Liver disorders such as auto-immune liver disease, isolated hypertransaminasaemia, non-alcoholic fatty liver disease and an association with hepatitis $\mathrm{C}$ virus infection have been reported in patients with coeliac disease. ${ }^{[5]}$ The onset of diarrhoea in our patient coincided with the deterioration in her liver function tests. We could not exactly determine whether this acute deterioration was in addition to her background of pre-existing HT1 or was due to coeliac hepatopathy.

Approximately $97 \%$ of individuals with coeliac disease have genetic markers on chromosome $6 \mathrm{p} 21,{ }^{[6]}$ while the gene for the FAH enzyme of HT1 is mapped on chromosome $15 .^{[1]}$ To the best of our knowledge, there is no literature reporting such an association between these two distinct diseases.

\section{Conclusion}

This is believed to be the first report of a child with HT1 associated with coeliac disease. We emphasise that any case with chronic diarrhoea should be promptly investigated for common potential causes.

\section{Declaration. None.}

\section{Acknowledgements. None.}

Author contributions. NAY conceptualised and designed the study, collected data, drafted the initial manuscript, and approved the final manuscript as submitted. EAM conceptualised the study, drafted the initial manuscript, and approved the final manuscript as submitted. HE- $\mathrm{K}$ designed the data collection instruments, co-ordinated and supervised data collection, critically reviewed the manuscript, and approved the final manuscript as submitted.

Funding. None.

Conflicts of interest. None.

1. Bergeron A, Astous MD, Timm DE, Tanguay RM. Structural and functional analysis of missense mutations in fumarylacetoacetate hydrolase, the gene deficient in hereditary tyrosinemia Type 1. J Biol Chem 2001;276(18):1522515231. http://www.jbc.org/content/276/18/15225.long

2. Nakamura K, Matsumoto S, Mitsubuchi H, Endo F. Tyrosinemia Type I. In: Lang F, ed. Encyclopedia of Molecular Mechanisms of Disease. Berlin: Springerlink; 2009:213-225. https://doi.org/10.1007/978-3-540-29676-8

3. Schiller LR, Pardi DS, Sellin JH. Chronic diarrhea: Diagnosis and management. Clin Gastroenterol Hepatol 2017;15:(2):182-193. https://doi.org/10.1016/j. cgh.2016.07.028

4. Santra S, Preece MA, Hulton SA, et al. Renal tubular function in children with tyrosinaemia type I treated with nitisinone. J Inherit Metab Dis 2008;31(3):399402. https://doi.org/10.1007/s10545-008-0817-x

5. Zali MR, Nejad MR, Rostami K, Alavian SM. Liver complications in celiac disease. Hepat Mon 2011;11(5):333-341.

6. Wolters V, Wijmenga C. Genetic background of celiac disease and its clinical implications. Am J Gastroenterol 2008;103(1):190-195. https://doi. org/10.1111/j.1572-0241.2007.01471.x

Accepted 31 July 2019. 